\title{
Parametric Equations of the Theory of Formation of Spherical Micelles
}

\author{
D.S.Grebenkov' \\ St. Petersburg State University, Department of Statistical Physics \\ ul. Ul'yanovskaya 1, Petrodvorets, 198904, Russia \\ Ecole Polytechnique, Laboratoire de Physique de la Matière Condensée \\ 91128 Palaiseau Cedex, France
}

\begin{abstract}
Using the notion of aggregation work, we construct a system of differential equations for the aggregation number of micelles which is a function of the parameters of micellization (parametric equations). There are explicit solutions for two important models of spherical micelles. Based on these solutions, we obtain an analytical expression for the equilibrium concentration of surfactant monomers and consequently for the whole spectrum of equilibrium concentrations of molecular aggregates in this framework. Accuracy of these expressions is discussed, and they are applied on an example of micelles formed by sodium dodecyl sulfate.
\end{abstract}

\section{Introduction}

The process of micellization is an important and interesting problem, whose mechanism is not yet understood. Its complexity does not allow to describe it completely. If the concentration $n_{1}$ of surfactant monomers (amphiphiles) exceeds the critical micellization concentration, the monomers can form aggregates called micelles. There are different structures of micelles (spherical, cylindrical, disk-like, inverse, etc., see (11)), and they are classified by $n_{1}$. For a relatively small concentration $n_{1}$, the spherical structure is the most favorable 2 . There are two essential advantages in this case. First, the concentration of monomers is small and therefore one can apply some useful approximations. Second, the geometry of micelles is described by the only parameter - the radius of the sphere. Moreover, this radius can be expressed in terms of aggregation number (the number of monomers which form an aggregate). Consequently, it is natural to use methods of the nucleation theory and, particularly, the notion of aggregation work. One can find its description and applications in [2]-[6] that investigate thermodynamic properties of micelles.

We propose a method which allows to determine the dependence of the equilibrium concentration of monomers and micelles on the essential parameters defining the process of micellization. We are going to establish parametric

\footnotetext{
${ }^{1}$ E-mail: Denis.Grebenkov@polytechnique.fr

${ }^{2}$ Here we will not consider the supplemental conditions which can be imposed by packing constraints, see 1 .
} 
equations which can be solved explicitly for the different model expressions of the aggregation work. Therefore, we will able to determine equilibrium concentration of monomers as a function of external parameters (e.g., temperature) in the framework of a given model. Consequently, it will allow to relate different models of spherical micelles among themselves and to compare them with regard to the experimental data.

The first part is devoted to introduction of the basic notions and the essential assumptions which will be used to establish parametric equations in the second part. The third and the fourth parts give solutions of these equations for the drop model and Grinin's model of spherical micelles. The final part describes some consequences and the possible generalizations of this formalism.

\section{Basic notions and essential assumptions}

Nucleation theory is based upon the notion of aggregation work $F_{\nu}$, i.e. minimal work required to form an aggregate from $\nu$ surfactant monomers (amphiphiles), where $\nu$ is referred to aggregation number. Function $F_{\nu}$ essentially defines the dynamics of the system, and completely determines equilibrium distribution of aggregates $n_{\nu}$ by virtue of the aggregation number $\nu$ according to Boltzmann's law,

$$
n_{\nu}=n_{1} e^{-F_{\nu}},
$$

where $n_{1}$ is the concentration of monomers ${ }^{3}$. Note that $F_{1}=0$ because the monomers already exist. Function $F_{\nu}$ depends on the aggregation number $\nu$, concentration of monomers in solution $n_{1}$, and parameters defining the micellization (e.g. temperature, molecular properties of solvent, structure of monomers, etc) which are denoted as $a_{1} \ldots a_{K}$. Note that the aggregation work $F_{\nu}$ does not depend on the concentrations $n_{\nu}(\nu>1)$. Indeed, we are working with spherical micelles, thus the concentration of monomers is relatively small and highly dominates the concentrations of dimers, trimers, etc. Hence, we can assume that aggregates are formed due to gradual attachment/detachment of monomers. In other words, we neglect the possibilities of attachment/detachment of dimers, trimers, etc. to the aggregate. Moreover, for the small concentration (i.e. the dilute solution) the dependence of function $F_{\nu}$ on $n_{1}$ is simply

\footnotetext{
${ }^{3}$ We shall use dimensionless quantities. Particularly, all the concentrations are expressed in convenient units: for example, in units of the critical micellization concentration; the aggregation work is measured in units of $k T$, where $k$ is Boltzmann constant, $T$ is absolute temperature.

${ }^{4}$ This is a direct consequence of the differential form of $F_{\nu}$ as difference of chemical potentials

$$
d F_{\nu}=\left(\mu_{\nu}-\mu_{0}\right) d \nu
$$

where $\mu_{\nu}$ is the chemical potential of any surfactant "inside" the aggregate consisting of $\nu$ molecules. Obviously, $\mu_{\nu}$ is independent of the concentration of monomers in the solution and depends only on the internal structure of aggregate. $\mu_{0}$ is the chemical potential of any surfactant in the solution. For the dilute solutions there is a simple expression for $\mu_{0}$

$$
\mu_{0}=\ln n_{1}+\chi(T),
$$

where $\chi(T)$ is a certain function of temperature (see 朋).
} 


$$
F_{\nu}=G_{\nu}-(\nu-1) \ln n_{1},
$$

where new function $G_{\nu}$ does not depend at all on the concentrations and is defined only by the parameters $a_{1} . . a_{K}$ and $\nu$. The condition $F_{1}=0$ implies $G_{1}=0$.

We make an important assumption of the closure of the system, i.e. the total number of amphiphiles (in monomers and aggregates) $N$ is fixed. This is an essential condition because one can imagine some processes where this number changes. From now on, $N$ will be a fixed external parameter. In this case, the law of mass conservation can be written as

$$
N=\sum_{\nu=1}^{\infty} \nu n_{\nu}
$$

Note that in real physical problems the summation is usually limited by a certain value $\nu_{m}$. However, it is convenient to use this notation, remembering that the finite sum can be "complemented by zeros" (which is equivalent to rapid growth of $F_{\nu}$ with increase of $\nu$ ). For the convergence of series it is sufficient that $F_{\nu}$ tends to infinity when $\nu \rightarrow \infty$, or that $G_{\nu}$ increases faster than a linear function of $\nu$.

As the mechanism of micelle formation is very complex, it is reasonable to use model expressions for $G_{\nu}$ which should represent the essential properties of micellization. We represent function $G_{\nu}$ as a sum of the fractional powers of $\nu$

$$
G_{\nu}=\sum_{m=0}^{M} c_{m} \nu^{\rho m}
$$

where $\rho>0$ is a constant for the particular model $\sqrt{6}$, the coefficients $c_{m}$ of this decomposition depend on the parameters $a_{1} \ldots a_{K}$, and there are only positive powers in the sum. Note that function $G_{\nu}$ has a physical sense only for natural numbers $\nu$. However, the formulae (14) allows us to interpolate $G_{\nu}$ for any real number $\nu \geq 1$. Obviously, one can imagine different interpolations but we shall use the simple on 8 given by (㺼). Furthermore, we shall differentiate some quantities by $\nu$, etc.

The important statement of the nucleation theory is following: every minimum of aggregation work corresponds to a stable (or quasi-stable) state of the

\footnotetext{
5 This representation is quite reasonable: for example, in the problem of condensation of vapor the aggregation work is the sum of the powers of $\nu^{1 / 3}$, in Grinin's model of spherical micelles - the sum of the powers of $\nu^{1 / 2}$.

${ }^{6}$ As we use the model expressions, we can think of $\rho$ as a rational number. If the sum (4) includes the linear term, we can take $\rho=1 / m_{0}$ with a natural number $m_{0}$.

7 The negative powers of $\nu$ in the decomposition have no practical use because we are interested in the quantity $G_{\nu}$ for large $\nu$

8 Nevertheless, sometimes it is convenient to change a little this canonical interpolation. Indeed, the limit of $G_{\nu}$ when $\nu \rightarrow 1$ is equal to $c=\sum_{m=0}^{M} c_{m}$, and nothing demands that it equals to zero (for example, see the drop model of spherical micelles, part 3). Therefore, we can change the expression (4) on the interval $(1,2)$ to obtain $\lim _{\nu \rightarrow 1} G_{\nu}=0$. The simple way is to add function $-c / \nu^{x}$ to $G_{\nu}$ where $x$ is a large positive number. We are interested in function $G_{\nu}$ for large $\nu$ thus the addition $-c / \nu^{x}$ has no any influence on our results.
} 
system of aggregates. We are interested in the formation of micelles, therefore there should be a "deep" minimum of $F_{\nu}$ (potential well). Moreover, this minimum is assumed to be unique and it will be denoted as $\nu_{s}$. Indeed, we are working with a small concentration of monomers, and consequently, there is only one stable state - spherical micelles. Using the relation (2), one can write the equation on $\nu_{s}$ as

$$
0=\frac{\partial F_{\nu}}{\partial \nu}\left(\nu_{s}\right)=\frac{\partial G_{\nu}}{\partial \nu}\left(\nu_{s}\right)-\ln n_{1}
$$

Physically, the quantity $\nu_{s}$ is the aggregation number of micelles, $\nu_{s} \gg 1$. In the practically important case the number of amphiphiles in micelles is comparable (or more) to the concentration of monomers.

Using all previous assumptions we can establish our formalism which defines the dependence of equilibrium concentration of monomers on the parameters of micellizaton.

\section{Parametric equations of the theory of forma- tion of spherical micelles}

Let us introduce a set of functions $f_{\alpha}(x)$ assuming $x>0$,

$$
f_{\alpha}(x)=\sum_{\nu=1}^{\infty} \nu^{\alpha} e^{-G_{\nu}} x^{\nu}=\sum_{\nu=1}^{\infty} e^{-h(\nu)},
$$

where new function $h(\nu)$ is

$$
h(\nu)=G_{\nu}-\nu \ln x-\alpha \ln \nu .
$$

There are three important properties :

1) All functions $f_{\alpha}(x)$ are defined for any positive $x$ because of a rapid growth of function $h(\nu)$ (since $G_{\nu}$ increases faster than $\nu$ ). Moreover, $f_{\alpha}(x) \geq 0$ is true for any $x>0$.

2) Simple verification shows that

$$
x \frac{\partial f_{\alpha}(x)}{\partial x}=f_{\alpha+1}(x) .
$$

Hereof it follows that all $f_{\alpha}(x)$ are smooth monotonously growing functions, with derivatives of all orders.

3) For $\alpha \ll \nu_{s}$ function $h(\nu)$ has a minimum which is with high accuracy equal to $\nu_{s}$.

Let us represent $f_{\alpha}(x)$ as a sum of three terms which correspond to monomers, small aggregates and micelles,

$$
f_{\alpha}(x)=x+\sum_{\nu=2}^{\nu_{0}} e^{-h(\nu)}+\sum_{\nu=\nu_{0}}^{\infty} e^{-h(\nu)}=x+f_{\alpha}^{0}(x)+\tilde{f}_{\alpha}(x) .
$$

\footnotetext{
9 The equation for the minimum of $h(\nu)$ is derived by subtracting $\alpha / \nu$ in the right-hand side of the equation (5). Under condition $\alpha \ll \nu_{s}$ this term can be neglected by comparison with the positive powers of $\nu$.
} 
It is necessary to say that the choice of this decomposition is artificial: $\nu_{0}$ depends on the given model. Usually $\nu_{0}$ can be chosen as the maximum of $F_{\nu}$, i.e. a point where $n_{\nu}$ has its minimum. As we take interest in formation of micelles, it is reasonable to suppose that the contribution of micelles dominates over the contributions of small aggregates,

$$
f_{\alpha}^{0}(x) \ll \tilde{f}_{\alpha}(x) .
$$

We neglect functions $f_{\alpha}^{0}(x)$, and the sum (9) becomes

$$
f_{\alpha}(x) \approx x+\tilde{f}_{\alpha}(x) .
$$

Now we calculate an approximation to function $\tilde{f}_{\alpha}(x)$ (which corresponds to micelles) for small values of $\alpha$. For this purpose we represent the corresponding sum in (9) as an integral, decompose $h(\nu)$ in the vicinity of its minimum $\nu_{s}$ up to the square terms, and calculate the obtained gaussian integral after replacing the lower integral limit by $-\infty$,

$$
\tilde{f}_{\alpha}(x) \approx \exp [-\beta(x)], \quad \beta(x)=h\left(\nu_{s}\right)+\frac{1}{2} \ln h^{\prime \prime}\left(\nu_{s}\right)-\frac{1}{2} \ln (2 \pi) .
$$

Due to the independence of $\nu_{s}$ of $\alpha$, we obtain an important relation between functions $\tilde{f}_{\alpha}$,

$$
\tilde{f}_{\alpha} / \tilde{f}_{\beta}=\left(\nu_{s}\right)^{\alpha-\beta}
$$

whence we conclude

$$
\tilde{f}_{\alpha}=\left(\nu_{s}\right)^{\alpha-1} \tilde{f}_{1} .
$$

In terms of functions $f_{\alpha}(x)$ the law of mass conservation (3) can be written as equation for $n_{1}$,

$$
f_{1}(x)=N \text {. }
$$

Due to the continuity and monotone growth of $f_{\alpha}(x)$, for any $N$ there is unique solution of (14) which is equal to $n_{1}$,

$$
f_{1}\left(n_{1}\right)=N \text {. }
$$

Substituting (12) and (15) in (11), we obtain the transcendental equation for the equilibrium concentration of monomers $n_{1}$ (cf. [5]),

$$
N=n_{1}+\exp \left[-\beta\left(n_{1}\right)\right]
$$

Practically this equation leads to the same difficulties in solution as the initial equation (15). Therefore we continue to develop our method.

Let us consider the equation (14). Once this equation is solved, we shall find the dependence of $x$ (i.e. $n_{1}$ ) on the parameters $a_{1} \ldots a_{K}$. One can treat this dependence as a trajectory in the phase space based on parameters $a_{1} \ldots a_{K}$, where function $f_{1}(x)$ is "integral of motion" (i.e. it is a constant on this trajectory). Fixing all parameters except one and differentiating (14) by this single parameter $a_{k}$, we express the derivatives $d x / d a_{k}$ according to 


$$
\frac{d x}{d a_{k}}=-\left[\frac{\partial f_{1}}{\partial x}\right]^{-1} \frac{\partial f_{1}}{\partial a_{k}}
$$

The derivative in denominator can be found with the help of (8). Now we calculate the derivative in nominator,

$$
\frac{\partial f_{1}}{\partial a_{k}}=\sum_{\nu=1}^{\infty} \nu e^{-G_{\nu}} x^{\nu}\left(-\frac{\partial G_{\nu}}{\partial a_{k}}\right)
$$

Taking into account the representation of $G_{\nu}$ as a sum of fractional powers of $\nu$, one has

$$
\frac{\partial G_{\nu}}{\partial a_{k}}=\sum_{m} c_{m, k} \nu^{\rho m}, \quad c_{m, k}=\frac{\partial c_{m}}{\partial a_{k}} .
$$

Collecting the previous expressions together, dividing by $x$ and bringing it under the sign of differential, we obtain

$$
\frac{d \ln x}{d a_{k}}=\frac{1}{f_{2}(x)} \sum_{m} c_{m, k} f_{\rho m+1} .
$$

Now we represent functions $f_{\alpha}$ according to (9), where all the terms $\tilde{f}_{\alpha}$ are expressed by (13) and all the terms $f_{\alpha}^{0}(x)$ are neglected by virtue of (10),

$$
\frac{d \ln x}{d a_{k}}=\frac{1}{x+\nu_{s} \tilde{f}_{1}}\left(x \sum_{m} c_{m, k}+\tilde{f}_{1} \sum_{m} c_{m, k} \nu_{s}^{\rho m}\right) .
$$

In the first part we demanded that the number of amphiphiles in micelles $\left(\tilde{f}_{1}\right)$ has the same or much greater order than in monomers $\left(n_{1}\right)$, therefore we can neglect $10 x$ and $x \sum_{m} c_{m, k}$ by comparison with $\nu_{s} \tilde{f}_{1}$ and $\tilde{f}_{1} \sum_{m} c_{m, k} \nu_{s}^{\rho m}$ in nominator and denominator respectively. We have

$$
\frac{d \ln x}{d a_{k}}=\sum_{m} c_{m, k} \nu_{s}^{\rho m-1}
$$

With equation (5) we obtain a closed system of equations for two unknown functions : $\ln x$ and $\nu_{s}$. Differentiating the equation (5) by $a_{k}$,

$$
\frac{d \ln x}{d a_{k}}=\rho \sum_{m} m\left(c_{m, k} \nu_{s}^{\rho m-1}+c_{m}(\rho m-1) \nu_{s}^{\rho m-2} \frac{d \nu_{s}}{d a_{k}}\right),
$$

we establish one differential equation for $\nu_{s}$. After simplification, this equation gets a form

$$
\frac{d \nu_{s}}{d a_{k}}=-\frac{\sum_{m} c_{m, k}(\rho m-1) \nu_{s}^{\rho m}}{\sum_{m} c_{m} \rho m(\rho m-1) \nu_{s}^{\rho m-1}}, \quad(k=1 . . K) .
$$

So, we have obtained a set of equations which define the dependence of the aggregation number of micelles $\nu_{s}$ on the parameters of the theory. We shall

\footnotetext{
${ }^{10}$ Here we make second approximation, after the gaussian one in calculation of $\tilde{f}_{\alpha}$. The essential simplification brought by this operation is the independence of derivatives $d \ln x / d a_{k}$ of $\tilde{f}_{1}$.
} 
call these equations parametric. Its solution is connected with the equilibrium concentration of monomers $n_{1}$ via (5)).

If we are interested only in dependence on one specific parameter $a_{k}$, it is sufficient to solve one of the equations (20) for this parameter. However, in this case there appears an arbitrary function of the other parameters. On the contrary, if we search for a complete solution, it is necessary to solve all the equations (20) and to accommodate all appearing arbitrary functions. Note that in the case of a large number of parameters this algorithm can be very complicated.

We make another important remark. If one adds an arbitrary function $g(\nu)$ to the expression of $G_{\nu}$ which does not depend on the set of parameters $a_{1} \ldots a_{K}$ and does not break a convergence of the series (16), then the equation (20) does not change. Indeed, function $g(\nu)$ disappears after differentiation by the parameters $a_{1} . . a_{K}$. Consequently, $g(\nu)$ has no influence ${ }^{\text {T1] }}$ on a solution of the parametric equations (20). On the contrary, the addition of $g(\nu)$ affects the equation (5), and the expression of equilibrium concentration of monomers will change according to the formula

$$
n_{1}^{\prime}=n_{1} \exp \left[-g^{\prime}\left(\nu_{s}\right)\right],
$$

where $n_{1}$ is a solution of (20) without $g(\nu)$. This simple proposition is very useful from the practical point of view. We see that the characteristic $\nu_{s}$ is universal, its equation (20) is independent of a small variations of function $G_{\nu}$. On the contrary, the equilibrium concentrations of monomers and micelles depend on the derivative of $g(\nu)$ according to (21).

The common property of the set of equations (20) is their independence of the linear term $\nu$ which disappears by virtue of a factor $(\rho m-1)$ when $m$ equals to $m_{0}=1 / \rho$ corresponding to a linear term (in the case when $1 / \rho$ is not natural number, a priori there is no linear term). Nevertheless, sometimes 12 there is a dependence of the equation (20) on the coefficient $c_{m_{0}}$ by virtue of the term $c_{0}$ which can contain this coefficient. However, usually one can neglect the term $c_{0}$ by comparison with $\nu_{s}^{\rho m}\left(\nu_{s} \gg 1\right)$. Note that every case should be analyzed particularly.

What is the qualitative information that is contained in the equation (20)? It seems possible to use this equation (and the whole method) to verify the following important hypothesis in the theory of micellization. As it was mentioned above, the exact expression for the aggregation work is unknown, therefore we use the model expressions (see, for example, (31)). However, it is natural to suppose that all physically measured quantities depend on global characteristics of the aggregation work (such as minimum of $F_{\nu}$, depth of this minimum, height of activation barrier, etc), rather than on a choice of its particular expression. If this hypothesis works, it is sufficient to take a simple expression for $F_{\nu}$ satisfying to the qualitative requests and to solve the problems with the use of this expression.

\footnotetext{
11 It is more accurate to say that function $g(\nu)$ has no influence on the analytic form of solution, but it changes, of course, initial conditions, and consequently, some constants of solution.

12 See, for example, Grinin's model, part 4.
} 


\section{Solution of the parametric equations for the drop model of spherical micelles.}

Let us consider a drop model of spherical micelles, firstly elaborated by Israelachvili, Mitchell and Niham (see [8]) and reformulated independently by Rusanov in terms of the aggregation work (see [9]). The basic ideas of this model are simple :

1) hydrophilic electrically charged heads form a surface of micelle (sphere);

2) hydrophobic tails are very flexible, and they form a liquid drop in the micellar core.

There are three terms in the aggregation work:

- bulk term (proportional to the number $\nu$ of amphiphiles in the aggregate);

- surface term corresponding to the attractive hydrophobic forces (and proportional to $s \nu$, where $s$ is the surface area per amphiphile; $s \nu \sim \nu^{2 / 3}$ for the spherical micelles) ;

- surface term corresponding to the repulsive electrostatic forces (proportional to $\nu / s \sim \nu^{4 / 3}$ as it has been shown by Tanford, [10]).

Leaving out the details, we write only the final expression for the aggregation work for this model,

$$
G_{\nu}=b_{1} \nu^{4 / 3}-\left(\frac{4}{3} \sqrt{2 b_{1} b_{3}}\right) \nu+b_{3} \nu^{2 / 3},
$$

where the parameters $\sqrt{13} b_{1}$ and $b_{3}$ are defined through the physical characteristics,

$$
b_{1}=\frac{(e z)^{2} \delta}{8 \pi \varepsilon_{0} \varepsilon \lambda^{2} k T}, \quad b_{3}=\frac{4 \pi \lambda^{2} \gamma_{0}}{k T},
$$

where $\lambda=(3 v / 4 \pi)^{1 / 3}$ is radius of a sphere which has the same volume as one hydrocarbon chain, $e z$ is electric charge of one amphiphile, $\varepsilon_{0}$ is dielectric constant, $\varepsilon$ is dielectric permittivity, $\delta$ is separation of the capacitor planes, $\gamma_{0}$ is surface tension of the hydrocarbon-water interface.

The expression 22 defines the coefficients of decomposition of the aggregation work by the powers of $\nu$ with $\rho=1 / 3$,

$$
c_{0}=0, \quad c_{1}=0, \quad c_{2}=b_{3}, \quad c_{3}=-\frac{4}{3} \sqrt{2 b_{1} b_{3}}, \quad c_{4}=b_{1} .
$$

In the framework of this model the parametric equations $(20)$ can be solved exactly, without any approximation (however, the parametric equations themselves remain approximate ones). Substituting the values of $c_{m}$ into the equations (20), we obtain

$$
\begin{aligned}
\frac{d \nu_{s}}{d b_{1}} & =-\frac{3}{2} \frac{\nu_{s}^{4 / 3}}{2 b_{1} \nu_{s}^{1 / 3}-b_{3} \nu_{s}^{-1 / 3}}, \\
\frac{d \nu_{s}}{d b_{3}} & =\frac{3}{2} \frac{\nu_{s}^{2 / 3}}{2 b_{1} \nu_{s}^{1 / 3}-b_{3} \nu_{s}^{-1 / 3}} .
\end{aligned}
$$

\footnotetext{
${ }^{13}$ We use Rusanov's notations.
} 
The substitution

$$
\nu_{s}=\left(b_{3} / b_{1}\right)^{3 / 2} \varphi^{3}\left(b_{1}, b_{3}\right)
$$

leads to

$$
2 b_{1} \frac{d \varphi}{d b_{1}}=\frac{\varphi\left(\varphi^{2}-1\right)}{2 \varphi^{2}-1}, \quad-b_{3} \frac{d \varphi}{d b_{3}}=\frac{\varphi\left(\varphi^{2}-1\right)}{2 \varphi^{2}-1},
$$

and we obtain the solution

$$
\begin{aligned}
\varphi^{2}\left(\varphi^{2}-1\right) & =\gamma_{3}\left(b_{3}\right) b_{1}, \\
\varphi^{2}\left(\varphi^{2}-1\right) & =\gamma_{1}\left(b_{1}\right) b_{3}^{-2} .
\end{aligned}
$$

Equaling the right-hand sides, we relate functions $\gamma_{1}\left(b_{1}\right)$ and $\gamma_{3}\left(b_{3}\right)$,

$$
\gamma_{1}\left(b_{1}\right) b_{1}^{-1}=\gamma_{3}\left(b_{3}\right) b_{3}^{2} .
$$

As the parameters $b_{1}$ and $b_{3}$ are independent, both sides of this equality are constant, whence

$$
\gamma_{1}\left(b_{1}\right)=\gamma(N) b_{1}, \quad \gamma_{3}\left(b_{3}\right)=\gamma(N) b_{3}^{-2},
$$

where a constant $\gamma(N)$ can be determined numerically for any $N$ (see table 1). Substituting function $\gamma_{1}\left(b_{1}\right)$ in the equation (26), one finds the dependence of the aggregation number of micelles $\nu_{s}$ on the parameters of micellization,

$$
\nu_{s}=\left(\alpha b_{3} / 2 b_{1}\right)^{3 / 2}, \quad \alpha=1+\sqrt{1+4 \gamma(N) b_{1} b_{3}^{-2}} .
$$

The equilibrium concentration of monomers can be found by substitution of (28) in the equation (5),

$$
n_{1}=\exp \left(\frac{2}{3} \sqrt{2 b_{1} b_{3}}\left[\alpha^{1 / 4}-\alpha^{-1 / 4}\right]^{2}\right) .
$$

Now it is useful to evaluate the values of parameters $b_{1}$ and $b_{3}$. We can obtain ${ }^{14}$

$$
b_{1} \approx 2, \quad b_{3} \approx 30
$$

for sodium dodecyl sulfate $\left(\mathrm{C}_{12} \mathrm{H}_{25} \mathrm{OSO}_{3} \mathrm{Na}\right)$ in water at the ambient temperature $(T=300 K)$ using characteristic values of the following parameters (see [1], 8]) :

- volume of the hydrocarbon tail of an amphiphile, $v=0.350 \mathrm{~nm}^{3}$;

- interfacial free energy per unit area of aggregate, $\gamma_{0}=50 \mathrm{erg} / \mathrm{cm}^{2}$;

- optimal surface area per amphiphile, $a_{0} \approx 0.62 \mathrm{~nm}^{2}$ (this value was used to calculate $b_{1}$, see for details [1]).

We have determined the dependence of equilibrium concentration of monomers on the parameters $b_{1}$ and $b_{3}$. The dependence on the total concentration of amphiphiles $N$ is contained in the constant $\gamma(N)$ which can be numerically

\footnotetext{
14 Note that these estimations are quite enough for our purposes. Indeed, we are working with the simple model, therefore we can not expect a high accuracy of predicted results.
} 
calculated for any $N$. Indeed, it is sufficient to find $n_{1}^{0}$ for one set of $b_{1}^{0}, b_{3}^{0}$ and $N$ by solving the equation (3) or (15), then to substitute the value of $n_{1}^{0}$ in the expression (29) and to express the constant $\gamma$. With the help of numerical simulations we obtain the values of $\gamma(N)$ for some $N$ represented in the table 1 .

\begin{tabular}{|c|c|c|c|c|c|c|c|}
\hline$N$ & 2.0 & 2.5 & 3.0 & 3.5 & 4.0 & 4.5 & 5.0 \\
\hline$n_{1}$ & 1.9865 & 2.1159 & 2.1471 & 2.1648 & 2.1771 & 2.1865 & 2.1942 \\
\hline$\gamma$ & -32.774 & -22.852 & -20.486 & -19.156 & -18.232 & -17.525 & -16.953 \\
\hline
\end{tabular}

Table 1. The values of constant $\gamma(N)$ and of equilibrium concentration of monomers $n_{1}$ as functions of $N$. For these simulations we have used $b_{1}=2.0$, $b_{3}=30$ corresponding to sodium dodecyl sulfate in water.

Note that the aggregation work $F_{\nu}$ for this model grows rapidly for small $\nu$; thus dimers, trimers and other small aggregates are completely negligible. Consequently, the number of amphiphiles in micelles can be well approximated as $N-n_{1}$.

The essential advantage of the expression (29) is its explicit form which allows to analyze dependencies of all the equilibrium concentrations on parameters $b_{1}$ and $b_{3}$. Moreover, this formula has a quite high accuracy. The table 2 represents its relative errors (in per cent) when parameters $b_{1}$ and $b_{3}$ are widely varied.

\begin{tabular}{|c|c|c|c|c|c|c|c|c|c|c|c|c|}
\hline$b_{3} \backslash b_{1}$ & 1.0 & 1.5 & 1.6 & 1.7 & 1.8 & 1.9 & 2.0 & 2.1 & 2.2 & 2.3 & 2.4 & 2.5 \\
\hline 28 & 0.9 & 0.5 & 0.3 & 0.1 & 0.0 & 0.3 & 0.5 & 0.7 & 1.0 & 1.3 & 1.6 & 1.9 \\
29 & 0.9 & 0.6 & 0.4 & 0.3 & 0.1 & 0.0 & 0.2 & 0.4 & 0.7 & 0.9 & 1.2 & 1.5 \\
30 & 0.9 & 0.7 & 0.6 & 0.4 & 0.3 & 0.2 & 0 & 0.2 & 0.4 & 0.9 & 0.8 & 1.0 \\
31 & 0.9 & 0.7 & 0.7 & 0.6 & 0.5 & 0.3 & 0.2 & 0.1 & 0.1 & 0.6 & 0.4 & 0.6 \\
32 & 0.9 & 0.8 & 0.7 & 0.7 & 0.6 & 0.5 & 0.4 & 0.3 & 0.2 & 0.0 & 0.1 & 0.2 \\
\hline
\end{tabular}

Table 2. The relative errors (in per cent) of the formula (29) when $b_{1}$ changes from 1.0 to 2.5, $b_{3}$ changes from 28 to 32 . Generally, these errors are less than $1 \%$ (beside of the right upper corner marked in italic that is not physical, see below).

Note that the variation of $b_{3}$ from 30 to 32 (28) can be caused by decreasing (increasing) the temperature by $20^{\circ} \mathrm{C}$ (i.e. the domain of variation of temperature is between $5^{\circ} \mathrm{C}$ and $45^{\circ} \mathrm{C}$ ). On the contrary, the parameter $b_{1}$ can be varied in the wide enough region. Indeed, if one adds salts (for example, $\mathrm{NaCl}$ ) in solution, they will screen electrostatic repulsion of amphiphile heads, i.e. the parameter $b_{1}$ will be decreased. Normally, it is difficult to increase $b_{1}$ considerably, thus the right upper corner of the table 2 is not physical. We can conclude that the formula (29) approximates the equilibrium concentration of monomers with relative errors less than $1 \%$. 


\section{Solution of the parametric equations for Grinin's model of spherical micelles.}

There is another model of spherical micelles elaborated by Grinin 12]. The hydrophobic tails of amphiphiles are assumed to be enough ordered in the core of micelles (on the contrary, for the drop model we assumed that hydrophobic tails are completely flexible as a liquid). The geometric restrains imply that the hydrophobic contribution to chemical potential of one amphiphile in aggregate is proportional to $\nu^{1 / 2}$. The hydrophilic effects can be described by the term proportional to $\nu$ (see [11]). The more detailed description can be found in the original article [12].

For our purposes the final expression for the aggregation work is important,

$$
G_{\nu}=\frac{b}{2}\left(\nu^{2}-1\right)-\frac{2 a}{3}\left(\nu^{3 / 2}-1\right)+\frac{a^{2}}{4 b}(\nu-1),
$$

the two parameters ${ }^{15}$ are : $a_{1}=a$ and $a_{2}=b$. The expression (31) is the decomposition of the aggregation work by the powers of $\nu$ with $\rho=1 / 2$ and with the coefficients

$$
c_{0}=\frac{2 a}{3}-\frac{b}{2}-\frac{a^{2}}{4 b}, \quad c_{1}=0, \quad c_{2}=\frac{a^{2}}{4 b}, \quad c_{3}=-\frac{2 a}{3}, \quad c_{4}=\frac{b}{2} .
$$

After calculating matrix of derivatives $c_{m, k}$ and substituting it in (20), we obtain two equations,

$$
\begin{aligned}
\frac{d \nu_{s}}{d a} & =\frac{2}{3} \frac{\nu_{s}^{3 / 2}}{2 b \nu_{s}-a \nu_{s}^{1 / 2}}, \\
\frac{d \nu_{s}}{d b} & =-\frac{\nu_{s}^{2}}{2 b \nu_{s}-a \nu_{s}^{1 / 2}}
\end{aligned}
$$

(in the nominator we have neglected a small term $(a / 2 b-2 / 3)$ by comparison with $\nu_{s}^{3 / 2}$ and $\nu_{s}^{2}$ ). The substitution

$$
\nu_{s}=(a / b)^{2} \varphi^{2}(a, b)
$$

reduces these equations to the quadratures

$$
-\frac{2 d a}{a}=\frac{d \varphi(2 \varphi-1)}{\varphi(\varphi-2 / 3)}, \quad \frac{3 d b}{2 b}=\frac{d \varphi(2 \varphi-1)}{\varphi(\varphi-2 / 3)},
$$

${ }^{15}$ As for the drop model, the parameters $a$ and $b$ are not physical, but they are connected with real physical characteristics,

$$
a=B \frac{d+d_{H_{2} O}-s^{1 / 2}}{(4 \pi)^{1 / 2} l_{c}}, \quad b=\frac{(z e)^{2}}{4 \pi \varepsilon_{0} \varepsilon l_{c} k T}\left[\left(n_{c}+r / l_{c}\right)^{-1}-\left(n_{c}+r / l_{c}+\delta / l_{c}\right)^{-1}\right],
$$

where $B$ is empiric constant (which equals to 1.4 under normal conditions, see [9]), $d$ is minimal distance between hydrophobic tail and water molecule, $d_{H_{2} O}$ is characteristic size of water molecule, $l_{c}$ is hydrocarbon length, $s$ is effective surface area of hydrophobic tail, $\delta$ is characteristic scale of space distribution of charge formed by aggregate, $e z$ is effective charge of hydrophilic head of amphiphile, $\varepsilon_{0}$ is dielectric constant, $\varepsilon$ is dielectric permittivity of solution, $n_{c}$ is number of carbon atoms in hydrophobic tail. 
which give after integration two equations of fourth degree connecting $\varphi$ with $a$ and $b$,

$$
\begin{aligned}
\varphi^{3}|\varphi-2 / 3| & =\gamma_{1}(b) a^{-4} \\
\varphi^{3}|\varphi-2 / 3| & =\gamma_{2}(a) b^{3},
\end{aligned}
$$

where $\gamma_{1}(b), \gamma_{2}(a)$ are arbitrary functions. Equaling the right-hand sides, one obtains : $\gamma_{1}(b)=C b^{3}, \gamma_{2}(a)=C a^{-4}$, i.e. the equation is

$$
\varphi^{3}|\varphi-2 / 3|=C a^{-4} b^{3},
$$

with an arbitrary constant $C$. Using small variations from the initial parameters, one can conclude that the factor $\varphi^{3}$ changes a little ${ }^{16}$. Then we obtain an approximate solution

$$
\varphi=2 / 3+\left(C a_{0}^{4} b_{0}^{-3}\right) a^{-4} b^{3},
$$

or, with the help of (34), we have

$$
\nu_{s}=\left(2 a / 3 b+\gamma_{G}(N) a^{-3} b^{2}\right)^{2},
$$

where a constant $\gamma_{G}$ can be defined for any $N$, and once defined (by the numerical solution of initial equation (3) for certain fixed parameters $a_{0}, b_{0}$ ), it becomes universal characteristic for the model under consideration. In the table 3 we show the values of $\gamma_{G}(N)$ for some $N$. Now one can use the equation (5) to calculate $n_{1}$,

$$
n_{1}=\exp \left[\frac{a^{2}}{36 b}\left(1+\gamma_{G}(N) a^{-4} b^{3}\right)^{2}\right] .
$$

Thus we have found the complete dependence of the equilibrium concentration of monomers on the parameters for Grinin's model of spherical micelles.

Note that the characteristic values of the parameters $a$ and $b$ of Grinin's model of spherical micelles are

$$
a \approx 1.5, \quad b \approx 0.1
$$

With the help of numerical simulations, we calculate the values of the constant $\gamma_{G}$ for some $N$ for these characteristic values of the parameters $a$ and $b$, see table 3 .

\footnotetext{
16 Indeed, using an explicit expression 31 for function $G_{\nu}$ we obtain an equation for extrema,$$
b \nu-a \nu^{1 / 2}+a^{2} / 4 b=\ln n_{1},
$$

with larger solution (corresponding to the minimum $\nu_{s}$ )

$$
\nu_{s}=\left(a / 2 b+\sqrt{\left(\ln n_{1}\right) / b}\right)^{2},
$$

whence it follows that $\varphi \geq 1 / 2$, therefore all changes occur in the vicinity of the point $2 / 3$.
} 


\begin{tabular}{|c|c|c|c|c|c|c|c|}
\hline$N$ & 2.0 & 2.5 & 3.0 & 3.5 & 4.0 & 4.5 & 5.0 \\
\hline$n_{1}$ & 1.6280 & 1.6460 & 1.6549 & 1.6608 & 1.6652 & 1.6687 & 1.6716 \\
\hline$\gamma_{G}$ & -592.08 & -541.82 & -517.62 & -501.66 & -489.76 & -480.29 & -472.42 \\
\hline
\end{tabular}

Table 3. Grinin's model: values of the constant $\gamma_{G}$ and of the equilibrium concentration of monomers $n_{1}$ for some $N$ calculated with the parameters $a=1.5$, $b=0.1$.

The table 4 contain ${ }^{17}$ the relative errors (in per cent) of the expression (38).

\begin{tabular}{|c|c|c|c|c|c|c|c|c|c|c|c|}
\hline$b \backslash a$ & 1.0 & 1.1 & 1.2 & 1.3 & 1.4 & 1.5 & 1.6 & 1.7 & 1.8 & 1.9 & 2.0 \\
\hline 0.05 & 0.0 & 0.9 & - & - & - & - & - & - & - & - & - \\
0.06 & 0.7 & 0.0 & 0.8 & - & - & - & - & - & - & - & - \\
0.07 & 1.2 & 0.8 & 0.1 & 0.8 & - & - & - & - & - & - & - \\
0.08 & 0.9 & 1.3 & 0.9 & 0.1 & 0.8 & - & - & - & - & - & - \\
0.09 & 1.3 & 1.2 & 1.4 & 0.9 & 0.0 & 0.9 & - & - & - & - & - \\
0.10 & - & 0.2 & 1.4 & 1.4 & 0.8 & 0 & 1.0 & - & - & - & - \\
0.11 & - & - & 0.6 & 1.6 & 1.4 & 0.7 & 0.1 & 1.2 & - & - & - \\
0.12 & - & - & - & 1.1 & 1.5 & 1.3 & 0.6 & 0.3 & 1.5 & - & - \\
0.13 & - & - & - & 0.4 & 1.5 & 1.7 & 1.3 & 0.5 & 0.5 & 1.8 & - \\
0.14 & - & - & - & - & 0.5 & 1.7 & 1.7 & 1.1 & 0.3 & 0.7 & 2.2 \\
0.15 & - & - & - & - & - & 1.1 & 1.8 & 1.6 & 1.0 & 0.0 & 1.0 \\
\hline
\end{tabular}

Table 4. Relative errors (in per cent) of the expression (38) calculated for a wide domain of the parameters $a$ and $b(N=2)$.

The absence of the values in the left low corner is caused by the condition $n_{1}>1$ (otherwise there are no stable aggregates). The right upper corner is empty by virtue of the condition $n_{1}<N$ (the opposite case has no physical sense). We see that the relative error of the approximate solution (38) changes in an irregular way, however, it does not exceed $2 \%$ when the parameters $a$ and $b$ take all physically reasonable values. This accuracy can be considered as a high one, thus we conclude that the formula (38) is a good approximation for the equilibrium concentration of monomers in Grinin's model. Nevertheless, there are problems which demand more accurate results. For this reason we would like to stress that the form of our expressions is explicit, therefore it is possible to analyze the results qualitatively and to compare different models.

Note that the form of the solution (38) is rather different from drop's one (29). However, we can not compare them directly because the parameters $a$, $b$ and $b_{1}, b_{3}$ are completely incompatibles. Thus, one should express these parameters in terms of the physical quantities (such as the global characteristics of the aggregation work), substitute their into (29) and (38), and after that to compare the results.

\footnotetext{
17 To fill this table, the equilibrium concentration of monomers $n_{1}$ for some values of parameters $a$ and $b$ was calculated by two different methods: by numerical solution of the equation (15) and with the help of the approximate formula (38).
} 


\section{Conclusions}

Based on the nucleation theory, we have developed the method which allows to obtain the parametric equations under rather general assumptions :

- closure of the system ;

- dilute solution of surfactants ;

- general model expression of $G_{\nu}$ as a sum of the fractional powers of $\nu$;

- comparability of the numbers of amphiphiles in monomers and micelles.

To deduce the parametric equations we have used these assumptions, but they are of the rather different significance. More important hypothesis is the closure of system. Some other ones are necessary to be mathematically rigorous. Finally, there are certain conditions which are used to specify the differential equations, to obtain concrete results (such as the solutions (29) or (38)), but they are not strictly necessary for the method's work. For example, the decomposition of function $G_{\nu}$ by the fractional powers of $\nu$ is physically reasonable and it simplifies the method significantly. But this assumption can be diminished, i.e. the expression of $G_{\nu}$ can be generalized. In other words, by concretizing the form of some expressions (for example, (2), (44), etc.), we simplify mathematically our problem, physically leaving it in general form.

We have obtained the differential equation for the important characteristic of micellization - for the aggregation number of micelles. Taking into account the remark in second part about the invariance of the parametric equations by addition of some function $g(\nu)$ to the aggregation work, we can conclude that $\nu_{s}$ is an universal quantity of micellization. On the contrary, the equilibrium concentrations of monomers and micelles essentially depend on the concrete expression $G_{\nu}$, consequently, they are not universal.

The system of parametric equations was solved for two practically important model of spherical micelles : the drop and Grinin's models. We have obtained an explicit dependence of the equilibrium concentration of monomers on the parameters of micellization. The numerical simulations showed that these formulae have a high accuracy.

At last, there appears a real possibility to verify the hypothesis that the physically measured quantities of micellization (e.g., the relaxation times) are independent of the concrete analytical expression of the aggregation work, but they are defined by its global characteristic (such as point of minimum, height of activation barrier, etc). If this hypothesis will be confirmed, it will justify the use of the simple model expressions of the aggregation work (such as (22) for the drop model, or (31) for Grinin's model). Otherwise, our method allows to compare different model expressions and the solutions based on them, and to determine the substantiality of the model and its accuracy.

We can conclude that elaborated method is effective enough both for applications (for example, to determine the dependencies of equilibrium concentration of monomers on the parameters for the two models of spherical micelles) and for the general analysis. We hope that it will allow to solve some problems in the theory of micelles, and to comprehend profoundly the mechanism of micellization. 


\section{Acknowledgement}

The author would like to thank Professor A.P.Grinin whose invaluable advice helped to develop the present method.

\section{References}

[1] Israelachvili J.M. Intermolecular and Surface Forces, Academic Press, $2^{\text {nd }}$ edition (1992).

[2] Rusanov A.I., Kuni F.M., Shekin A.K. Thermodynamic and Kinetic Foundations of the Theory of Micellization, Colloid Journal, V.62, N2, p.199 (2000).

[3] Kuni F.M., Grinin A.P., Shekin A.K., Rusanov A.I. Thermodynamic and Kinetic Foundations of the Theory of Micellization. 2. Direct and Reverse Fluxes of Molecular Aggregates over the Activation Barrier of Micellization, Colloid Journal, V.62, N2, p.204 (2000).

[4] Kuni F.M., Grinin A.P., Shekin A.K., Rusanov A.I. Thermodynamic and Kinetic Foundations of the Theory of Micellization. 3. Initial Stages of Micellization. Colloid Journal, V.64, N4, p.505 (2000).

[5] Kuni F.M., Grinin A.P., Shekin A.K., Rusanov A.I. Thermodynamic and Kinetic Foundations of the Theory of Micellization. 4. Kinetics of Establishing the Equilibrium in Micellar Solution, Colloid Journal (2000). (in print)

[6] Kuni F.M., Rusanov A.I., Grinin A.P., Shekin A.K. Thermodynamic and Kinetic Foundations of the Theory of Micellization. 5. Hierarchy of Kinetic Times, Colloid Journal (2000). (in print)

[7] Landau L.D., Lifshits E.M. Statistical Physics, part 1 (1980).

[8] Israelachvili J.N., Mitchell D.J., Niham B.W. Theory of Self-Assembly of Hydrocarbon Amphiphiles into Micelles and Bilayers, J.Chem. Soc. Farad. Trans. II, 72, p.1525 (1976).

[9] Rusanov A.I. Micellization in Solutions of Surfactants, SPb, Khimiya, 280 pp. (1992).

[10] The Hydrophobic Effect: Formation of Micelles and Biological Membranes, C.Tanford, J.Wiley and Son Eds, New York (1980).

[11] Rusanov A.I. Thermodynamics of Ionic Micelles, Uspekhi Khimii, V.58, N.2. pp.169-195 (1989).

[12] Grinin A.P. Model of Accounting of Hydrophobic and Hydrophilic Effects in Thermodynamics of Spherical Micelles with Moderate Aggregation Numbers, Vestnik Sankt-Peterburgskogo Universiteta, Ser.4, vol.1 (N4) pp. 3-9 (1996). 